\title{
Interval Analysis for Thermodynamic Calculations in Process Design: A Novel and Completely Reliable Approach
}

\author{
James Z. Hua* \\ Department of Chemical Engineering \\ University of Illinois at Urbana-Champaign \\ Urbana, IL 61801 USA \\ Robert W. Maier, Stephen R. Tessier ${ }^{\dagger}$, Joan F. Brennecke \\ and Mark A. Stadtherr \\ Department of Chemical Engineering \\ University of Notre Dame \\ Notre Dame, IN 46556 USA
}

8th International Conference on Properties and

Phase Equilibria for Product and Process Design,

Noordwijkerhout, The Netherlands, April 26-May 1, 1998

Keywords: Method of Calculation, Vapor-Liquid Equilibria, Liquid-Liquid Equilibria, Azeotropes, Interval Analysis

(revised, October, 1998)

*currently at Shell Chemical Company, Houston, TX, USA

${ }^{\dagger}$ Currently at AlliedSignal Inc., Troy, MI, USA

${ }^{\ddagger}$ Author to whom all correspondence should be addressed. Phone: (219)631-9318; Fax: (219)6318366; E-mail: markst@nd.edu 


\begin{abstract}
Recently, a robust new computational technique, based on interval analysis, has been developed for solving the difficult nonlinear problems arising in the modeling of phase behavior. This technique can be used, with mathematical and computational guarantees of certainty, to find the global optimum of a nonlinear function or to enclose any and all roots of a system of nonlinear equations. As shown in the applications here to phase stability analysis and to the location of homogeneous azeotropes, it provides a method that can guarantee that the correct result is found, thus eliminating computational problems that may potentially be encountered with currently available techniques. The method is initialization independent; it is also model independent, straightforward to use, and can be applied in connection with any equation of state or activity coefficient model.
\end{abstract}




\section{Introduction}

Computational problems such as convergence to a trivial or incorrect root, or to a local rather than global optimum, or failure to converge to a physically feasible solution at all [e.g., 1], are not uncommon difficulties in thermodynamic calculations. Since, in the modeling and design of separation processes, such failures can be critical, many techniques have been developed in attempts to provide increased reliability. However, until now, there has been no general purpose technique that could provide complete reliability for computations of phase behavior. In this paper we review recent work on the development and use of a robust new computational technique, based on interval analysis, for solving the difficult nonlinear problems arising in thermodynamics. This technique can be used, with mathematical and computational guarantees of certainty, to find the global optimum of a nonlinear function or to find (more precisely to enclose, as discussed in [2]) any and all roots of a system of nonlinear equations. While this technique has the potential to be applied to a wide variety of thermodynamic calculations important in chemical process design, in this paper we will concentrate on phase stability analysis, using both cubic equation of state models and excess Gibbs energy models, and on the location

of homogeneous azeotropes. Since the technique is model independent and completely general, its use can be readily extended to any thermodynamic model in any equilibrium situation. 


\section{Methodology}

\subsection{Interval Computations}

A real interval, $X$, is defined as the set of real numbers lying between (and including) given upper and lower bounds; i.e., $X=[a, b]=\{x \in \Re \mid a \leq x \leq b\}$. A real interval vector $\mathbf{X}=\left(X_{1}, X_{2}, \ldots, X_{n}\right)^{T}$ has $n$ real interval components and can be interpreted geometrically as an $n$-dimensional rectangle. Note that in this section lower case quantities are real numbers and upper case quantities are intervals. Several good introductions to computation with intervals are available $[3,4,5]$.

Of particular interest here are interval Newton/generalized bisection (IN/GB) methods. These techniques provide the power to find, with confidence, enclosures of all solutions of a system of nonlinear equations $[3,5]$, and to find with total reliability the global minimum of a nonlinear objective function [4], provided only that upper and lower bounds are available for all variables.

For a system of nonlinear equations $\mathbf{f}(\mathbf{x})=\mathbf{0}$ with $\mathbf{x} \in \mathbf{X}^{(0)}$, the basic iteration step in interval Newton methods is, given an interval $\mathbf{X}^{(k)}$, to solve the linear interval equation system

$$
F^{\prime}\left(\mathbf{X}^{(k)}\right)\left(\mathbf{N}^{(k)}-\mathbf{x}^{(k)}\right)=-\mathbf{f}\left(\mathbf{x}^{(k)}\right)
$$

for a new interval $\mathbf{N}^{(k)}$, where $k$ is an iteration counter, $F^{\prime}\left(\mathbf{X}^{(k)}\right)$ is an interval extension of the Jacobian of $\mathbf{f}(\mathbf{x})$ over the current interval $\mathbf{X}^{(k)}$, and $\mathbf{x}^{(k)}$ is a point in the interior of $\mathbf{X}^{(k)}$. The interval extension $\mathbf{F}\left(\mathbf{X}^{(k)}\right)$ of a real function $\mathbf{f}(\mathbf{x})$ over an interval $\mathbf{X}^{(k)}$ is an enclosure of the range of the function over that interval; that is $\mathbf{F}\left(\mathbf{X}^{(k)}\right) \supseteq\left\{\mathbf{f}(\mathbf{x}) \mid \mathbf{x} \in \mathbf{X}^{(k)}\right\}$. An interval extension can be computed by substituting interval quantities for the corresponding 
real quantities and using interval arithmetic, or in other ways. In the interval extension of the Jacobian, each matrix element is an interval extension of the corresponding element in the real Jacobian. It can be shown that any root $\mathbf{x}^{*} \in \mathbf{X}^{(k)}$ is also contained in the image $\mathbf{N}^{(k)}$, suggesting the iteration scheme $\mathbf{X}^{(k+1)}=\mathbf{X}^{(k)} \cap \mathbf{N}^{(k)}$. While this iteration scheme can be used to tightly enclose a solution, what is also of significance here is the power of Eq. (1) as an existence and uniqueness test. It can be proven (e.g., [3,5]) that if $\mathbf{N}^{(k)} \subset \mathbf{X}^{(k)}$, then there is a unique zero of $\mathbf{f}(\mathbf{x})$ in $\mathbf{X}^{(k)}$, and that Newton's method with real arithmetic can be used to find it, starting from any point in $\mathbf{X}^{(k)}$. This suggests a root inclusion test for $\mathbf{X}^{(k)}$ :

1. (Range Test) Compute an interval extension $\mathbf{F}\left(\mathbf{X}^{(k)}\right)$ containing the range of $\mathbf{f}(\mathbf{x})$ over $\mathbf{X}^{(k)}$ and test to see whether it contains zero. Clearly, if $0 \notin \mathbf{F}\left(\mathbf{X}^{(k)}\right) \supseteq\left\{\mathbf{f}(\mathbf{x}) \mid \mathbf{x} \in \mathbf{X}^{(k)}\right\}$ then there can be no solution of $\mathbf{f}(\mathbf{x})=\mathbf{0}$ in $\mathbf{X}^{(k)}$ and this interval need not be further tested.

2. (Interval Newton Test) Compute the image $\mathbf{N}^{(k)}$ by solving Eq. (1).

(a) If $\mathbf{X}^{(k)} \cap \mathbf{N}^{(k)}=\emptyset$, then there is no root in $\mathbf{X}^{(k)}$.

(b) If $\mathbf{N}^{(k)} \subset \mathbf{X}^{(k)}$, then there is exactly one root in $\mathbf{X}^{(k)}$.

(c) If neither of the above is true, then no further conclusion can be drawn.

In the last case, one could then repeat the root inclusion test on the next interval Newton iterate $\mathbf{X}^{(k+1)}$, assuming it is sufficiently smaller than $\mathbf{X}^{(k)}$, or one could bisect $\mathbf{X}^{(k+1)}$ and repeat the root inclusion test on the resulting intervals. This is the basic idea of interval Newton/generalized bisection methods. A detailed step-by-step description of the basic IN/GB algorithm used here is given by Schnepper and Stadtherr [6], and additional details are provided 
by Hua et al. [2]. Our implementation of the IN/GB method for the phase stability problem is based on appropriately modified routines from the packages INTBIS [7] and INTLIB [8]. In this paper, we review the application of the interval method to solve problems in phase stability analysis and in locating homogeneous azeotropes.

\subsection{Phase Stability Analysis}

The determination of phase stability, i.e., whether or not a given mixture can split into multiple phases, is a key step in phase equilibrium calculations, and thus in the simulation and design of a wide variety of processes, especially those involving separation operations such as distillation and extraction. The determination of phase stability is often done using tangent plane analysis. A phase at specified temperature $T$, pressure $P$, and feed mole fraction $\mathbf{z}$ is unstable (in this context, unstable refers to both the metastable and classically unstable cases)

if the reduced molar Gibbs energy of mixing surface $m=\Delta \hat{G}_{m i x} / R T$ ever falls below a plane tangent to the surface at $\mathbf{z}$. That is, if the tangent plane distance

$$
D=m-m_{0}-\sum_{i=1}^{n}\left(\frac{\partial m}{\partial x_{i}}\right)_{0}\left(x_{i}-z_{i}\right)
$$

is negative for any composition $\mathbf{x}$, the phase is unstable. The subscript zero indicates evaluation at $\mathbf{x}=\mathbf{z}$, and $n$ is the number of components. A common approach for determining if $D$ is ever negative is to minimize $D$ subject to the mole fractions summing to one. It is readily shown that the stationary points in this optimization problem can be found by solving the system of nonlinear equations:

$$
\left[\left(\frac{\partial m}{\partial x_{i}}\right)-\left(\frac{\partial m}{\partial x_{n}}\right)\right]-\left[\left(\frac{\partial m}{\partial x_{i}}\right)-\left(\frac{\partial m}{\partial x_{n}}\right)\right]_{0}=0, \quad i=1, \ldots, n-1
$$




$$
1-\sum_{i=1}^{n} x_{i}=0
$$

If an equation of state model $\operatorname{EOS}(\mathbf{x}, v)=0$ is used to determine $m$, then the EOS provides an additional equation and an additional variable, the molar volume $v$.

The $n \times n$ system given by Eqs. (3)-(4) above, or the $(n+1) \times(n+1)$ system that results when an EOS model is used, has a trivial root at the feed composition and frequently has multiple nontrivial roots as well. Thus conventional equation solving techniques may fail by converging to the trivial root or give an incorrect answer to the phase stability problem by converging to a stationary point that is not the global minimum of $D$. Standard methods (e.g., [9]) for solving the phase stability problem typically rely on the use of multiple initial guesses. However, these methods offer no guarantee that the global minimum in the tangent plane distance has been found. Because of the difficulties that thus arise, there has been significant recent interest in the development of more reliable methods for solving the phase stability problem. For example, Sun and Seider [10] apply a homotopy-continuation method, which will often find all the stationary points, and is easier to initialize than Michelsen's approach. However, their technique is still initialization dependent and provides no theoretical guarantees that all stationary points have been found. McDonald and Floudas [11] show that for certain activity coefficient models, the phase stability problem can be reformulated to make it amenable to solution by powerful global optimization techniques. This method does provide a mathematical guarantee that the global minimum of the tangent plane distance has been found.

An alternative approach for solving the phase stability problem is to use the interval method described above to solve Eqs. (3)-(4) for the stationary points of $D$. This was originally sug- 
gested by Stadtherr et al. [12], who applied it in connection with activity coefficient models, as later done also by McKinnon et al. [13]. This technique, is initialization independent and can solve the phase stability problem with mathematical certainty, while also dealing automatically with rounding error. Recently Hua et al. [2,14,15] extended this method to problems modeled with cubic equation of state (EOS) models, in particular the Van der Waals (VDW), Peng-Robinson (PR) and Soave-Redlich-Kwong (SRK) models with standard mixing rules.

\subsection{Location of Homogeneous Azeotropes}

The determination of the existence and composition of homogeneous azeotropes is important both from both theoretical and practical standpoints in the analysis of phase behavior and in the synthesis and design of separation systems. The nonlinear form of the thermodynamic equations for phase equilibrium makes the computation of azeotropes a particularly difficult problem. It has thus attracted significant attention, as reviewed recently by Widagdo and Seider [16]. Fidkowski et al. [17] have presented a homotopy continuation method for this purpose. While this method is very reliable, a drawback is that it cannot guarantee that all azeotropes have been found. More recently, Harding et al. [18] have used a global optimization procedure, which, assuming no explicit temperature dependence in the liquid phase activity coefficient models, does provide a mathematical guarantee that all azeotropes have been enclosed. Maier et al. [19] have discussed how the interval method described above can be used to solve the homogeneous azeotrope problem, providing a mathematical and computational guarantee that all azeotropes have been enclosed, even in the case of explicit temperature dependence in the liquid phase activity coefficient models. 
At a homogeneous azeotrope, vapor and liquid phases of the same composition are in equilibrium. For a set of $k$ nonzero components in a system of $n$ components, with the assumptions of ideal vapor behavior and Poynting correction factors of one, and use of the azeotropy condition $x_{i}=y_{i}$, where $x_{i}$ and $y_{i}$ are the liquid and vapor phase compositions, respectively, the equilibrium condition (equifugacity equations) can be written as

$$
\ln P-\ln P_{i}^{s a t}(T)-\ln \gamma_{i}^{L}(T)=0, \quad \forall i \in \mathcal{C}_{n z}
$$

where $\mathcal{C}_{n z}$ is the set of $k$ nonzero components in question, and we have emphasized the temperature dependence of the pure component vapor pressures $P_{i}^{s a t}$ and liquid phase activity coefficients $\gamma_{i}^{L}$. This equation, and the constraint that the mole fractions sum to one constitute a $(k+1) \times(k+1)$ set of nonlinear equations that can be solved for azeotropic composition(s) and temperature(s). Solving this system, we will obtain all $k$-ary azeotropes for the chosen component set $\mathcal{C}_{n z}$. To find all the azeotropes in the overall $n$ component system, a sequence (unordered) of such systems must be solved, one for each combination of $k$ components, $k=2, \ldots, n$. These equation systems may have multiple roots or no roots at all. A solution method is required that is guaranteed to enclose all roots and that is also capable of verifying when none exist. The interval approach discussed above is very well suited to this difficult task [19].

To model the temperature dependence of $P_{i}^{s a t}(T)$ we use the Antoine equation. In many cases, the temperature dependence of $P_{i}^{s a t}(T)$ is significantly stronger than the temperature dependence of $\gamma_{i}^{L}(T)$, which arises only in the temperature dependence of the binary interaction parameters in the activity coefficient model used. Thus, as done by Harding et al. [18], it 
is not unreasonable to evaluate $\gamma_{i}^{L}(T)$ at some reference temperature $T_{R E F}$ and then treat it as independent of temperature, instead of using a fully temperature dependent $\gamma_{i}^{L}(T)$ model. Assuming a good guess of $T_{R E F}$ is made, this approach may provide good estimates for the azeotropes in the fully temperature dependent model. However, there is no guarantee of this, and it is possible that the number of azeotropes found in the $T_{R E F}$-based model will not be the same as the number of azeotropes that exist in the fully temperature dependent model, even if a relatively good guess of $T_{R E F}$ has been made [19]. Finally, it should be noted that because solutions of the equifugacity equations may not be stable phases (the liquid may split), any solutions enclosed should next be checked for phase stability, as described above.

\section{Results}

\subsection{Phase Stability}

Hua et al. $[2,14,15]$ have applied the interval approach to solve several phase stability problems using various cubic equation of state models. We consider two such problems here. The first, which has not been presented previously, involves the binary system of carbon dioxide and trans-2-hexen-1-ol. The second is a ternary system of nitrogen, methane and ethane. Stadtherr et al. [12] have also used the interval technique to solve phase stability problems for excess Gibbs energy models. One such problem, not presented previously, is considered here. It is the quaternary system of $n$-propanol, $n$-butanol, benzene and water.

Problem 1. This is a binary mixture composed of carbon dioxide (1) and trans-2-hexen-1-ol (2) at $303.15 \mathrm{~K}$ and 69.7016 bar. The PR model was used with parameters calculated from $T_{c 1}$ 
$=304.2 \mathrm{~K}, P_{c 1}=73.8 \mathrm{bar}, \omega_{1}=0.225, T_{c 2}=601.76 \mathrm{~K}, P_{c 2}=36.73$ bar, $\omega_{1}=0.7241$, and a binary interaction parameter $k_{12}=0.084$. These conditions are very close to a three-phase line. In such regions, conventional methods for determining phase stability are prone to difficulty. For example, on this problem, for the unstable feed of $z_{1}=0.9991$, Michelsen's vapor/liquid flash algorithm, as implemented in LNGFLASH from the IVC-SEP package, a code that in general we have found to be extremely reliable, incorrectly predicts that this mixture is stable. Results of using the interval approach for this problem are shown in Table 1. This shows that we are able to locate all five stationary points for this feed and find the global minimum of $D$ as being negative at -0.004239 , thus demonstrating instability. Two other pressures and feed compositions were also considered and correct solutions were efficiently found as shown in Table 1. It should be noted that the presence of multiple real volume roots at some conditions in this problem does not present any difficulty, since the solver simply finds enclosures of all roots for the given system. Thus, nothing needs to be done to select the right volume roots.

Problem 2. This is a mixture of nitrogen (1), methane (2) and ethane (3) at $270 \mathrm{~K}$ and 76 bar. The PR model was used with $T_{c 1}=126.2 \mathrm{~K}, P_{c 1}=33.9 \mathrm{bar}, \omega_{1}=0.04, T_{c 2}=190.6 \mathrm{~K}, P_{c 2}=46.0$ bar, $\omega_{2}=0.008, T_{c 3}=305.4 \mathrm{~K}, P_{c 3}=48.8 \mathrm{bar}, \omega_{3}=0.098$, and the binary interaction parameters $k_{12}=0.038, k_{13}=0.08$ and $k_{23}=0.021$. Four feeds were considered, with results shown in Table 2. The first two feeds are not stable and the other two feeds are stable. The second and third feeds represent particularly difficult problems, since they are in the vicinity of the critical point of the mixture, yet the interval algorithm has no problem efficiently determining the correct solutions.

Problem 3. This is a mixture of $n$-propanol(1), $n$-butanol(2), benzene(3) and water(4) at 
$P=1 \mathrm{~atm}$ and $T=298 \mathrm{~K}$ modeled using the NRTL equation. Five different feed compositions were tested using the interval method with the correct results efficiently obtained in each case, indicating the only the second feed is stable. The interval technique can be applied in connection with any activity coefficient or EOS model.

In general, four-component problems, such as this one, and binary and ternary problems, such as the previous two examples, can be routinely solved with good computational efficiency. However, as the number of components grows, the computational performance becomes less predictable, with some problems solved efficiently and others requiring unreasonable computation time. This is because, like any technique (e.g., branch and bound) based on a binary tree, the worst-case computational complexity of the method described is exponential. This does not necessarily mean that large problems cannot be solved efficiently (the simplex algorithm for linear programming also has a worst-case complexity that is exponential), but that excessive computation time requirements are a possibility when solving large problems. This is consistent with the previous experience of Schnepper and Stadtherr [6] in applying the interval method to process modeling problems. Some relatively large (over a hundred variables) problems could be efficiently solved, while other smaller problems required much greater computational effort.

\subsection{Homogeneous Azeotropes}

Maier et al. [19] applied the interval algorithm to the solution of several homogeneous azeotrope problems, involving from two to five components and using the Wilson, NRTL and UNIQUAC models with explicit temperature dependence. One such problem is considered here. This is a ternary system modeled using UNIQUAC and consisting of benzene, ethanol, 
and water at 1 atmosphere. Table 4 shows that the interval method can efficiently find all the homogeneous azeotropes in this system, and also identify when there is no homogeneous azeotrope. Experimentally, this ternary system is known to have homogeneous azeotropes for the benzene/ethanol and ethanol/water binary pairs, and no homogeneous ternary azeotrope. The computed results for this system match the experimental data well in all respects. However, our computed results differ from those of Harding et al. [18], in that they compute a ternary azeotrope, while we do not. Presumably, this is due to some difference in the model parameters used in [18]. It should be noted that for the benzene/water binary, there is a solution to Eq. (5), as indicated in Table 4. However, contrary to what was indicated in [19], this solution does not represent a homogeneous azeotrope, since the liquid phase will split. This emphasizes the need, discussed above, to check solutions for liquid phase stability.

\section{Concluding Remarks}

As seen in the applications here to phase stability analysis and to the location of homogeneous azeotropes, the interval technique described here can solve phase behavior problems with complete reliability, providing a method that can guarantee with mathematical certainty that the correct result is found, thus eliminating computational problems that may potentially be encountered with currently available techniques. The method is initialization independent; it is also model independent, straightforward to use, and can be applied in connection with any equation of state or with activity coefficient model. 
Acknowledgments - This work has been supported in part by the donors of The Petroleum Research Fund, administered by the ACS, under Grant 30421-AC9, by the National Science Foundation Grants CTS95-22835 DMI96-96110 and EEC97-00537-CRCD, by the Environmental Protection Agency Grant R824731-01-0, by the Department of Energy Grant DE-FG0796ER14691, and by a grant from Sun Microsystems, Inc.

\section{References}

[1] K. A. Green, S. Zhou and K. D. Luks, Fluid Phase Equilib., 84 (1993) 49-78.

[2] J. Z. Hua, J. F. Brennecke and M. A. Stadtherr, Ind. Eng. Chem. Res., 37 (1998) 1519-1527.

[3] A. Neumaier, Interval Methods for Systems of Equations, Cambridge University Press, Cambridge, England, 1990.

[4] E. R. Hansen, Global Optimization Using Interval Analysis, Marcel Dekkar, New York, 1992.

[5] R. B. Kearfott, Rigorous Global Search: Continuous Problems, Kluwer Academic Publishers, Dordrecht, The Netherlands, 1996.

[6] C. A. Schnepper and M. A. Stadtherr, Comput. Chem. Eng., 20 (1996) 187-199.

[7] R. B. Kearfott and M. Novoa, ACM Trans. Math. Software, 16 (1990) 152-157.

[8] R. B. Kearfott, M. Dawande, K.-S. Du and C.-Y. Hu, ACM Trans. Math. Software, 20 (1994) 447-459. 
[9] M. L. Michelsen, Fluid Phase Equilib., 9 (1982) 1-19.

[10] A. C. Sun and W. D. Seider, Fluid Phase Equilib., 103 (1995) 213-249.

[11] C. M. McDonald and C. A. Floudas, AIChE J., 41 (1995) 1798-1814.

[12] M. A. Stadtherr, C. A. Schnepper, J. F. Brennecke, AIChE Symp. Ser., 91:304 (1995) $356-359$.

[13] K. I. M. McKinnon, C. G. Millar and M. Mongeau, in C. A. Floudas and P. M. Pardalos (Eds.), State of the Art in Global Optimization: Computational Methods and Applications, Kluwer Academic Publishers, Dordrecht, The Netherlands, 1996, pp. 365-382.

[14] J. Z. Hua, J. F. Brennecke and M. A. Stadtherr, Fluid Phase Equilib., 116 (1996) 52-59.

[15] J. Z. Hua, J. F. Brennecke and M. A. Stadtherr, Comput. Chem. Eng., 22 (1998) 12071214.

[16] S. Widagdo and W. D. Seider, AIChE J., 42 (1996) 96-130.

[17] Z. T. Fidkowski, M. F. Malone and M. F. Doherty, Comput. Chem. Eng., 17 (1993) 11411155.

[18] S. T. Harding, C. D. Maranas, C. M. McDonald and C. A. Floudas, Ind. Eng. Chem. Res., 36 (1997) 160-178.

[19] R. W. Maier, J. F. Brennecke and M. A. Stadtherr, AIChE J., 44 (1998) 1745-1755. 


\section{Notation}

$\mathcal{C}_{n z}$, a set of nonzero components.

$D$, tangent plane distance function.

f, a real function vector.

$\mathbf{F}$, interval extension of $\mathbf{f}$.

$F^{\prime}$, interval extension of Jacobian of $\mathbf{f}$.

$m$, reduced Gibbs energy of mixing.

$n$, number of components.

$\mathbf{N}$, the interval-Newton image, an interval.

$P$, pressure.

$P^{s a t}$, vapor pressure.

$\Re$, the set of real numbers.

$T$, temperature.

$v$, molar volume

$x$, a real number (variable).

$\mathbf{x}$, a real vector; also composition vector.

$X$, an interval.

$\mathbf{X}$, an interval vector.

$\mathbf{z}$, feed composition vector.

0, used as subscript to indicate evaluation at feed composition.

$\gamma_{i}^{L}$, liquid-phase activity coefficient. 
Table 1: Problem 1: PR, carbon dioxide(1) and trans-2-hexen-1-ol(2).

\begin{tabular}{||c||c|c|c||}
\hline \hline $\begin{array}{c}\text { Feed } \\
\left(P, T, z_{1}, z_{2}\right)\end{array}$ & Roots & $D$ & CPU Time (s) \\
& $\left(x_{1}, x_{2}, v\right)$ & & Sun Ultra 2/1300 \\
\hline \hline$(69.7016,303.15$, & $(0.9991,0.0009,160.8)$ & 0.0 & 0.714 \\
$0.9991,0.0009)$ & $(0.9968,0.0032,106.1)$ & 0.0020 & \\
& $(0.9728,0.0272,61.59)$ & -0.004 & \\
& $(0.8428,0.1572,57.97)$ & 0.0030 & \\
\hline$(120.0,303.15$, & $(0.7018,0.2982,65.53)$ & $3.6 \times 10^{-4}$ & \\
$0.85,0.15)$ & $(0.7645,0.2355,60.86)$ & $-4.1 \times 10^{-4}$ & \\
& $(0.9200,0.0800,53.91)$ & $-4.7 \times 10^{-4}$ & \\
\hline$(80.0,303.15$, & $(0.7078,0.2922,69.99)$ & -0.003 & 0.709 \\
$0.85,0.15)$ & $(0.85,0.15,57.38)$ & 0.0 & \\
& $(0.9600,0.0400,57.76)$ & -0.004 & \\
\hline
\end{tabular}

Table 2: Problem 2: PR, $\mathrm{N}_{2}(1), \mathrm{CH}_{4}(2)$ and ethane (3), $P=76$ bar, $T=270 \mathrm{~K}$.

\begin{tabular}{||c||c|c|c||}
\hline \hline $\begin{array}{c}\text { Feed } \\
\left(P, T, z_{1}, z_{2}\right)\end{array}$ & $\begin{array}{c}\text { Roots } \\
\left(x_{1}, x_{2}, v\right)\end{array}$ & $\begin{array}{c}\text { CPU Time (s) } \\
\text { Sun Ultra 1/170 }\end{array}$ \\
\hline \hline$(0.30,0.10,0.60)$ & $(0.312,0.102,0.587,153)$ & $-5.8 \times 10^{-6}$ & 1.981 \\
& $(0.300,0.100,0.600,147)$ & 0.0 & \\
& $(0.133,0.068,0.799,77.5)$ & -0.0148 & \\
\hline$(0.15,0.30,0.55)$ & $(0.150,0.300,0.550,132)$ & 0.0 & 4.977 \\
& $(0.147,0.297,0.556,130)$ & $3.55 \times 10^{-7}$ & \\
& $(0.097,0.245,0.658,90.3)$ & -0.0012 & \\
\hline$(0.08,0.38,0.54)$ & $(0.080,0.380,0.540,120)$ & 0.0 & 3.626 \\
\hline$(0.05,0.05,0.90)$ & $(0.050,0.050,0.900,69.6)$ & 0.0 & 0.768 \\
\hline \hline
\end{tabular}


Table 3: Problem 3: NRTL, $n$-Propanol(1), $n$-Butanol(2), Benzene(3) and Water(4), $P=1$ atm, $T=298 \mathrm{~K}$.

\begin{tabular}{||c||c|c|c||}
\hline \hline & Number of & & CPU Time (s) \\
Stationary & & Sun Ultra \\
Feed $\left(z_{1}, z_{2}, z_{3}, z_{4}\right)$ & Points & $D_{\text {min }}$ & $2 / 1300$ \\
\hline \hline$(0.148,0.052,0.600,0.200)$ & 3 & -0.340 & 1.101 \\
\hline$(0.25,0.25,0.25,0.25)$ & 3 & 0.0 & 1.655 \\
\hline$(0.148,0.052,0.700,0.100)$ & 3 & -0.311 & 1.748 \\
\hline$(0.25,0.15,0.40,0.20)$ & 3 & -0.039 & 2.615 \\
\hline$(0.25,0.15,0.35,0.25)$ & 3 & -0.074 & 2.592 \\
\hline \hline
\end{tabular}

Table 4: Homogeneous azeotropes: UNIQUAC, Benzene(B), Ethanol(E) and Water(W), $P=$ $1.0 \mathrm{~atm}$. CPU time is on a Sun Ultra 1/140.

\begin{tabular}{||c||c|c|c||}
\hline \hline Components & Mole Fraction (B E W) & Temperature $\left({ }^{\circ} \mathrm{C}\right)$ & CPU Time (s) \\
\hline \hline $\mathrm{BE}$ & 0.5520 .4480 .000 & 67.66 & 0.036 \\
\hline $\mathrm{BW}$ & $(0.5720 .0000 .428)^{*}$ & $(61.98)^{*}$ & 0.037 \\
\hline $\mathrm{EW}$ & 0.0000 .8860 .115 & 78.11 & 0.041 \\
\hline $\mathrm{BEW}$ & no azeotrope & & 1.21 \\
\hline total & & & 1.32 \\
\hline \hline
\end{tabular}

${ }^{*}$ This is a solution to Eq. (5), but is not a homogeneous azeotrope, since the liquid phase will split. 